(C) 2006 IEEE. Personal use of this material is permitted. However, permission to reprint/republish this material for advertising or promotional purposes or for creating new collective works for resale or redistribution to servers or lists, or to reuse any copyrighted component of this work in other works must be obtained from the IEEE. 


\section{Comparison of Opportunistic Scheduling Policies in Time-Slotted AMC Wireless Networks}

\author{
Amoakoh Gyasi-Agyei \\ School of Advanced Technologies \& Processes \\ Central Queensland University \\ Rockhampton, QLD-4701, Australia \\ Email: gyasi-agyei@ieee.org
}

\author{
Seong-Lyun Kim \\ School of Electrical \& Electronic Engineering \\ Yonsei University \\ 134 Sinchon-Dong, Seodaemun-Gu, Seoul 120-749, Korea \\ Email: slkim@yonsei.ac.kr
}

\begin{abstract}
We consider scheduling schemes which exploit the random variability in wireless channels to maximize wireless throughput. We survey ten of such opportunistic scheduling policies, namely, PFS, OCASD, TAOS-1, I-OCASD, CASTI, EXP, $M-L W D F$, FIFO, RR and MaxC/I and compare them in terms of system throughput, fairness (user isolation) and the distributions of user starvation periods (packet delays) in a time-slotted wireless network like CDMA/HDR using adaptive modulation and coding (AMC). The results aids in determining the suitability of any of the ten policies in a wireless network depending on which metric is considered critical.
\end{abstract}

Keywords-Channel-aware scheduling, channel state dependent scheduling, cross-layer MAC design, multi-user diversity scheduling, radio resource management, wireless MAC.

\section{INTRODUCTION}

The proper functioning of every multi-access facility, such as wireless networks, requires a medium access coordination (MAC) scheme. MAC schemes for wireless networks can be broadly grouped into link/rate adaptation (LRA) based (traditional MAC) and modern opportunistic MAC (OMAC). MAC for all 2G wireless systems, such as GSM, IS-136 and IS-95, are based on LRA. We focus on OMAC in this article.

OMAC is the modern view of communicating over spatiotemporally varying wireless link whereby the multi-user diversity is exploited rather than combated to maximize bandwidth efficiency or system throughput. In this scenario the scheduler suspends transmitting data to a user at a poor link state (and hence low transmission rate) until its link hits near its peak or improves. Hence, in its traditional form, an OMAC seeks to pick among competing users the one who is currently experiencing the relatively best channel conditions in each scheduling instant. The chance that the OMAC finds a user whose link is close to its peak increases with the variability (randomness) of users' links and hence the number of users sharing the medium. OMAC research so far has focused on conventional cellular network architecture in which the network coverage area is divided into cells, each of which is served by a (centralized) controller. The controller (system) can be a single-carrier or multi-carrier. Usually, the system uses a single-slot TDM/TDMA transmission per RF carrier. That is, transmission time is slotted, and in each time slot only one out of the users competing for service in a cell (sector) is selected for scheduling per RF carrier. For a packet based system, usually only one packet (or part thereof) is transmitted in each scheduling epoch. Such a single-slot TDM/TDMA transmission can be used for both slotted-CDMA and pure TDMA networks. Mobiles measure the qualities on their forward links and feed back to the controller on a period basis. Hence, the time slot duration should be much less than the channel coherence time (which is inversely proportional to the Doppler frequency shift) for the feedback channel gains to be useful. Thus, on a fast fading channel where OMAC achieves its maximum multi-user diversity gains, the channel quality feedback should be very frequent. This can penalize system's signaling load. The OMAC decides which user to schedule in each time slot using an algorithm relating the current link quality of the selected user, among other things.

OMAC is anticipated to reduce transceiver complexity and hence reduce power dissipation and costs. As traffic is scheduled to/from a user only when its link hits near its peak, time to transmit the same amount of data can be reduced. This allows a device to remain longer in energy-conserving mode and prolong its energy lifetime. This is critical for devices whose energy supply cannot be easily replenished. The only major weakness identified so far in OMAC is the potential traffic delay violations resulting from delaying transmission until a user's link hits near its peak. This delay, referred to here as starvation period (SVP), is examined in this article.

As shown in Fig. 1, OMAC requires a cross-layer protocol design approach, where the estimated instantaneous channel quality indicator (CQI) is fed to the MAC layer from the physical layer, traffic QoS related information (TQI) is fed to MAC by higher layers such as network or application layer, and the data link layer performs its error management through MAC. The CQI feedback can be the estimated instantaneous carrier-to-interference ratios $\left(\mathrm{CIR}_{m}(t)\right)$, supportable data rates $\left(R_{m}(t)\right)$, received signal strength indications (RSSI), or bit error rates $\left(\mathrm{BER}_{m}(t)\right)$ of users' links. The TQI feedback can be traffic timing constraints or user/queue service history such as cumulated transported amount of traffic. Due to its cross-layer design nature, OMAC requires a wider spectrum of expertise on its designers. The cross-layer nature also embeds OMAC with the potential to revolutionize the design of wireless data networks from physical to networking layers. 


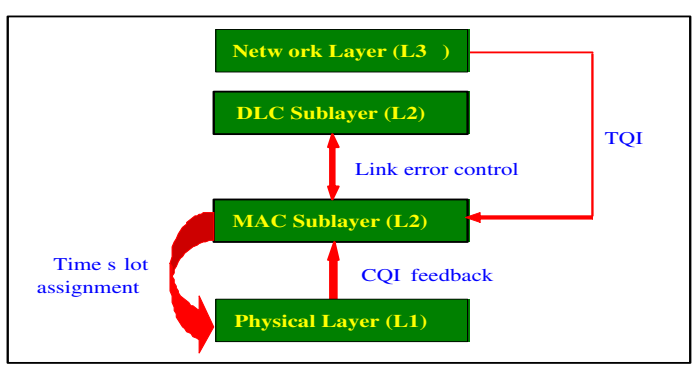

Fig. 1. Cross-layer view of opportunistic scheduling for wireless networks [CQI=channel quality indicator, TQI=traffic QoS related information].

All the OMAC schemes surveyed in this article can equally be used in commercial wireless system such as CDMA/HDR, although PFS is the default scheduler.

The rest of the article follows the pattern: we formulate the problem at hand in Section I-A and the article's contribution in Section I-B. The opportunistic scheduling schemes under study are surveyed in Section II. Simulations, numerical results and simulation assumptions are presented in Section III. The article concludes in Section IV.

\section{A. Problem Statement}

We have multiple mobile users, say $M$, competing for a shared wireless resource at a cell (or a sector of a cell) of a cellular communications network at a given time, $t$. Each of the users can be backlogged with traffic of two types simultaneously: real-time (RT) or non-real-time (NRT). RT packets have delay deadlines after which they become useless and thus declared lost. Hence, each traffic type requires different service level (QoS) from the network. Also, different mobile users are usually located at different spatial positions in the cell-some are closer to the serving base station, while others may be located at the cell boundary. At a given operating radio frequency, the channel variations across multiple mobile users also depend on other factors such as user mobility pattern and environmental clutter. Hence, different users experience different channel qualities in a given moment. This is referred to as multi-user diversity. Other assumptions used are:

1) Transmission time is slotted and only one user is scheduled at a given time. Once a user is picked for scheduling its entire head-of-line (HOL) packet is sent through over one or more contiguous time slots.

2) The scheduler has perfect knowledge of the updated estimated wireless channel states of users competing for service on a time slot basis.

3) The deadlines of RT packets are ordered in a nondecreasing order, with the HOL packet in a RT queue having the earliest deadline.

4) The user/queue which optimizes a non-pre-emptive priority value (see below) in a given scheduling epoch is picked for scheduling. Ties in any of the scheduling policies are broken randomly.

5) The wireless channel condition (and hence $R_{m}(t)$ ) for a user does not change for the duration of a packet transmission. For the time frame $W$ (known as latency time-scale or sliding scheduling window) the user population is assumed static and all users are continuously backlogged.

The task now is to design an optimum scheduler that assigns service order to multiple mobile users sharing a facility in a way that maximizes the overall system throughput while considering fairness and delay constraints.

\section{B. Our Contribution}

Our main contribution in this work include the definition of a new scheduling policy called CASTI (see Section IIC). Additionally, we survey and analyse the performance of several wireless scheduling schemes with respect to system throughput, fairness, packet delay distributions via the distributions of starvation periods (SVPs). An opportunistic scheduling has to temporarily suspend transmitting packet(s) to/from a user in poor channel state until its link quality improves relative to other users in order to maximize the overall system throughput. Hence, how much the service to a given user/queue can be delayed is relevant as it affects packet delay deadlines. Service suspension leads to starvation, and the SVP should not violate packet timing constraints to reduce packet losses in the network. Analyzing the SVP distribution of a scheduling scheme aids in determining its suitability to sustain time-sensitive network traffic with delay bounds. Wireless bandwidth is expensive and finite. Hence, bandwidth efficient radio resource management (RRM) schemes are relevant, the reason for system throughput considerations. Fair RRM schemes prevent resource hogging and service starvation to users/queues. An unfair RRM scheme can provoke churn of network users and cost service provider dollars.

\section{Opportunistic Scheduling Schemes}

Over the recent years opportunistic transmission scheduling for wireless networks has become a topic of intense research interest and several articles has been written on it. Such schedulers do not suffer from the hidden-terminal and exposed-terminal problems common to other multiple access coordination protocols. They exploit the random variability inherent in wireless channels to maximize overall system throughput (spectral efficiency). This is based on information theoretic principle called multi-user diversity, i.e. different users competing for the shared wireless channel undergo different channel qualities at the same time. In order to cater for fairness and timing issues of network traffic, various variations to 'pure' opportunistic scheduling have also been proposed. This section reviews only a sample of opportunistic transmission schedulers that are drawn upon in the work presented in this article. In the following $R_{m}(t)$ is the supportable data rate on user $m$ 's link at time $t$.

\section{A. OCASD Scheduler}

The Optimum Channel-Aware Scheduling with Service Differentiation (OCASD) [1], [2] is a traffic-aided OMAC which optimizes the trade-off between short-term fairness and 
throughput maximization. OCASD delivers superior throughput gains while offering short-term fairness and considering traffic delay constraints. However, OCASD does not guarantee that the desirable delay constraints of all packets are met. Hence, EXP/OCASD was proposed to provide a stricter delay constraints to improve service to delay-sensitive applications, such as VoIP, video/audio streaming and video/audio conferencing [3]. We refer to the vector $x=(i, m)$ as a connection. In the scheduling period starting at time $t$ OCASD picks the connection $x_{t}^{*}$ such that

$x_{t}^{*}=\arg \max _{x}\left\{\left[\left|w_{i}-\frac{B_{i}(t)}{B(t)}\right| \cdot B(t)\right]^{\operatorname{sgn}\left(w_{i}-\frac{B_{i}(t)}{B(t)}\right)} R_{m}(t) / d_{i}\right\}$,

where $w_{i}$ is the weight assigned to traffic class $i \in$ $\{R T, N R T\}, B(t)=\sum_{i \in \mathcal{B}} B_{i}(t), \mathcal{B}$ is the set of backlogged queues at time $t$, and the amount of traffic scheduled from/to traffic class $i$ so far is updated using the first-order IIR filter structure

$$
B_{i}(t+1)=(1-1 / W) B_{i}(t)+1 / W \times L_{i}(t) \delta(t-i) .
$$

The weight $w_{i}$ depends on the characteristics and hence the QoS requirements of traffic class $i . d_{i}$ is the delay deadline (or bound) of the HOL packet of size $L_{i}(t)$ in queue $i \in$ $\{R T, N R T\}$. Note that queue $i$ is penalized at time $t$ if $w_{i}<$ $\frac{B_{i}(t)}{B(t)}$ as in that case $\operatorname{sgn}\left(w_{i}-\frac{B_{i}(t)}{B(t)}\right)=-1$ and thus its priority scaling factor $(\cdot)^{\operatorname{sgn}\left(w_{i}-\frac{B_{i}(t)}{B(t)}\right)}$ is less than unity.

$W$ is the sliding time frame in which a given set of $M$ users share a finite wireless resource. The sliding time frame (aka latency time scale or scheduling window) $W$ is an important design parameter as it indicates the trade-off between throughput maximization, fairness and packet delay profile. The delay profile is crucial for RT traffic. $W$ determines how long a packet can be delayed before being scheduled. A longer $W$ is good for throughput maximization but it is limited by the maximum delay tolerance of network applications. A typical value proposed for most data applications is $100 \mathrm{~ms}$ [4]. The dirac delta impulse function is defined as

$$
\delta(t-k)= \begin{cases}1 & \text { if } t=k \\ 0 & \text { otherwise }\end{cases}
$$

$t=k$ means that user/queue $k$ is scheduled in time slot $t$.

\section{B. I-OCASD Scheduler}

The inverted OCASD (I-OCASD) [5] is an opportunistic scheduler which provides fairness while optmization wireless spectral efficiency. In the scheduling interval starting at time $t$ I-OCASD picks the connection $x_{t}^{*}$ such that

$$
x_{t}^{*}=\arg \min _{x} \quad B_{i}(t) / R_{m}(t),
$$

where $B_{i}(t)$ is defined as in (2).

\section{CASTI Scheduler}

The main difference between Channel-Aware Scheduling with Transmitted Traffic Information (CASTI) and OCASD [1], [2] is that the former assigns weights to users rather than traffic classes and there is no traffic class based queuing. Hence, $w_{m}$ is the weight assigned to user $m$. All other parameters are modified accordingly. In the scheduling period starting at time $t$ CASTI picks the user $m_{t}^{*}$ such that

$$
m_{t}^{*}=\arg \max _{m} U\left(w_{m}, B_{m}(t), R_{m}(t)\right),
$$

where

$$
U(\cdot)=\left[\left|w_{m}-\frac{B_{m}(t)}{B(t)}\right| \cdot B(t)\right]^{\operatorname{sgn}\left(w_{m}-\frac{B_{m}(t)}{B(t)}\right)} R_{m}(t),
$$

and $B(t)=\sum_{m=1}^{M} B_{m}(t)$. The average amount of traffic scheduled from user $m$ at time $t$ is updated as

$$
B_{m}(t+1)=(1-1 / W) B_{m}(t)+1 / W \times L_{m}(t) \delta(t-m)
$$

where $L_{m}(t)$ is the size of user $m$ 's HOL packet at time $t$. As $\operatorname{sgn}\left(w_{m}-f_{m}(t)\right) \in\{-1,0,+1\}$, user $m$ is penalised at time $t$ if it has received cumulative service exceeding its assigned weight $w_{m} \in(0,1]$ in $[0, t)$.

\section{Round-Robin Scheduler}

In round-robin (RR) scheduling arriving packets are queued by users, while the scheduler polls queues for service in a cyclic order irrespective of the wireless link conditions of the users to send/receive the packets. As greedy users flood their own queues, RR is able to provide user protection or isolation. By scheduling users/queues in a cyclic order, RR is able to provide fairness. Hence, it is used as fairness benchmark with respect to other schedulers. In the scheduling period starting at time $t$ RR picks user $m_{t}^{*}$ such that

$$
m_{t}^{*}=\operatorname{modulo}(t, M)+1
$$

where $1 \leq t \leq W$.

\section{E. MaxSNR Scheduler}

The maximum SNR (MaxSNR) scheduling picks a user $m^{*}$ among all active users in the system at time $t$ which has the best signal-to-noise ratio (SNR), or equivalently, the best feasible instantaneous data rate $R_{m}(t)$, i.e. select user $m^{*}$ that fulfills [6], [4]

$$
m_{t}^{*}=\arg \max _{m} R_{m}(t)=\arg \max _{m} C I R_{m}(t) .
$$

Note that SNR (or CIR) and data rate have a one-to-one mapping for a given channel bandwidth. Hence, this scheduler is also referred to as MaxC/I or Maximum C/I. The MaxC/I rule can easily starve users that undergo an extended period of poor link state, but provides very attractive system throughput. Hence, it can be used as throughput benchmark with respect to other scheduling policies. 


\section{F. PFS Scheduler}

Proportional fair sharing (PFS) scheduling [7], [8] is an OMAC scheme which provides fairness across users but not flows (or traffic classes). Hence, it cannot quantify service level of individual flows backlogged in a given user. At time $t$ PFS picks user $m^{*}$ among all backlogged users in the system which has the best feasible data rate normalized by the average throughput it has received so far, i.e.

$$
m_{t}^{*}=\arg \max _{m} R_{m}(t) / S_{m}(t)
$$

where the short-term mean data rate of user $m$ up to time $t$ is updated using the exponentially weighted low-pass filter

$$
S_{m}(t)=(1-1 / W) S_{m}(t-1)+1 / W \times R_{m}(t) \delta(t-m)
$$

The PFS is the default scheduler for the downlink of CDMA/HDR (aka 1xEV-DO, IS-856) which is an evolution of 3GPP2's cdma2000 sharing an RF space with IS-95.

\section{G. FIFO Scheduler}

The first in, first out (FIFO) scheduler is completely oblivious of wireless channel conditions, as its policy is purely based on how long a packet/user has been awaiting its service turn. FIFO is also referred to as first come, first served (FCFS). In the scheduling period starting at time $t$ the FIFO scheduler picks the user fulfilling

$$
m_{t}^{*}=\arg \max _{m} S V P_{m}(t)
$$

where $S V P_{m}(t)$ is the number of time slots that user $m$ has been waiting for service turn at time $t$, i.e. its starvation period. $S V P_{m}(t)$ is a congestion indication parameter at user (queue) $m$ at time $t$. Besides poor spectral efficiency, the FIFO scheduling does not provide fairness nor provide protection of behaving queues against resource-hogging users/queues. However, it can be used as latency benchmark with respect to other scheduling policies.

\section{H. M-LWDF Scheduler}

The priority value assigned to a user by the modified largest weighted delay first (M-LWDF) scheduler [9] is equal to that of the PFS policy scaled by the weighted delay the user has endured. In the scheduling interval starting at time $t$ the M-LWDF policy picks user $m_{t}^{*}$ among all competing users satisfying

$$
m_{t}^{*}=\arg \max _{m} a_{m} S V P_{m}(t) R_{m}(t) / S_{m}(t)
$$

where $S_{m}(t)$ is defined as in (11) and $a_{m}>0$ is a parameter indicating the QoS level desired by user $m$. $S_{m}(t)$ can also be the median of $R_{m}(t)$. We set $a_{m}=1, \forall m$ in this article.

\section{Exponential Scheduler}

The exponential scheduler (EXP) [10] attempts to equalize the weighted delays (starvation times) of all queues when their differences becomes large. It is designed to support real-time services in an AMC/TDM wireless system. In the scheduling period starting at time $t$ the EXP scheduler picks user $m_{t}^{*}$ such that

$$
m_{t}^{*}=\arg \max _{m} \frac{R_{m}(t)}{S_{m}(t)} \exp \left(\frac{a_{m} S V P_{m}(t)-\delta}{1+\sqrt{\delta}}\right)
$$

where $\delta=\frac{1}{M} \sum_{m} a_{m} S V P_{m}(t)$ and $M$ is the number of users competing for service simultaneously. $S V P_{m}(t)$ can also be the number of packets in user $m$ 's buffer at time $t$. As can be observed in (14), a large weighted delay $a_{m} S V P_{m}(t)$ overrides $R_{m}(t)$, hence restricting packet delays to a certain level. The EXP rule, however, reduces virtually to the PFS policy at low weighted delays. $S_{m}(t)$ is defined as in (11). We set $a_{m}=1, \forall m$ in this article.

\section{J. TAOS-1 Scheduler}

TAOS-1 (Traffic-Aided Opportunistic Scheduling) [11] is a heuristic opportunistic scheduling scheme that unifies file size information and wireless channel variations in order to reduce file completion time (i.e. waiting time plus transmission time) and/or the overall system completion time. TAOS has many variants but we consider here only TAOS-1. Let $X_{m}(t)$ be the size of user $m$ 's backlogged file at time $t$ and $F_{m}=X_{m}(t=$ $0)$ be its initial file size. At time $t$ TAOS- 1 picks user $m^{*}$ which fulfils

$$
m_{t}^{*}=\arg \min _{m} S_{m}(t) F_{m} / R_{m}(t)
$$

where $S_{m}(t)$ is updated as in (11). TAOS-1 assumes that each user has only one backlogged file at any time. In order to compare TAOS-1 with other schedulers, we set $F_{m}=L_{m}(t)$ in the simulations, where $L_{m}(t)$ is the size of the HOL packet in user $m$ 's queue at time $t$.

\section{Simulation And Numerical Results}

This section discusses the metrics used for the performance analyses, as well as the traffic and channel assumptions.

\section{A. Performance Metrics}

The performance metrics of interest here are per-user fairness, system throughput, and the distribution of user starvation period (SVP) or latency in an adaptive coding and modulation wireless system. We define the average throughput for connection $x$ up to time $t$ by the sample mean $\theta_{x}(t) \stackrel{\text { def }}{=}$ $\frac{1}{t} \sum_{l=0}^{t} R_{m^{*}}(l) \delta\left(x_{l}^{*}-x\right)$, where $m^{*}=x^{*}[2]$. Hence, the throughput for user $m$ up to time $t$ is

$$
\theta_{m}(t)=\frac{1}{t} \sum_{l=0}^{t} R_{m}(l) \delta\left(x_{l}^{*}[2]-m\right), m=1,2, \cdots, M
$$


TABLE I

CDMA/HDR SYSTEM PARAMETERS TO ACHIEVE 1\% PACKET ERROR RATE IN A 5-KM ISOLATED CELL WITH UNIFORM DISTRIBUTION OF USERS.

\begin{tabular}{|c|c|c|c|c|c|c|}
\hline $\begin{array}{l}\text { DRC } \\
\text { Index }\end{array}$ & $\begin{array}{l}\text { Modulation } \\
\text { Scheme }\end{array}$ & $\begin{array}{l}\text { Code } \\
\text { Rate }\end{array}$ & $\begin{array}{l}\text { Packet } \\
\text { Size } \\
{\left[L_{i}(t)\right.} \\
\text { bits] }\end{array}$ & $\begin{array}{l}\text { Data } \\
\text { Rate } \\
{\left[R_{m}(t)\right.} \\
\mathrm{kb} / \mathrm{s}]\end{array}$ & $\begin{array}{l}\text { Minimum } \\
\text { Required } \\
C / I(\mathrm{~dB})\end{array}$ & $\begin{array}{l}\text { Time } \\
\text { Slots } \\
\text { Needed } \\
\left(N_{t s}\right)\end{array}$ \\
\hline 1 & QPSK & $1 / 5$ & 1024 & 38.4 & -12.5 & 16 \\
\hline 2 & QPSK & $1 / 5$ & 1024 & 76.8 & -9.5 & 8 \\
\hline 3 & QPSK & $1 / 5$ & 1024 & 153.6 & -6.5 & 4 \\
\hline 4 & QPSK & $1 / 5$ & 1024 & 307.2 & -4.1 & 2 \\
\hline 5 & QPSK & $1 / 5$ & 2048 & $307.2 \mathrm{~L}$ & -0.9 & 4 \\
\hline 6 & QPSK & $1 / 3$ & 1024 & 614.4 & -4.0 & 1 \\
\hline 7 & QPSK & $1 / 3$ & 2048 & $614.4 \mathrm{~L}$ & -1.0 & 2 \\
\hline 8 & QPSK & $1 / 3$ & 3072 & 921.6 & 3.3 & 2 \\
\hline 9 & 7-PSK & $1 / 3$ & 2048 & 1228.8 & 1.3 & 1 \\
\hline 10 & 8-PSK & $1 / 3$ & 4096 & $1228.8 \mathrm{~L}$ & 7.2 & 2 \\
\hline 11 & 16-QAM & $1 / 3$ & 3072 & 1843.2 & 3.0 & 1 \\
\hline 12 & 16-QAM & $1 / 3$ & 4096 & 2457.6 & 9.5 & 1 \\
\hline
\end{tabular}

while the throughput for traffic class $i$ up to time $t$ is

$$
\theta_{i}(t)=\frac{1}{t} \sum_{l=0}^{t} R_{m}(l) \delta\left(x_{l}^{*}[1]-i\right), i \in\{R T, N R T\} .
$$

Thus the overall average system throughput over time $t$ is $\theta(t)=\sum_{i=1}^{c} \theta_{i}(t)=\sum_{m=1}^{M} \theta_{m}(t)$. Delay distribution is defined as $\operatorname{Pr}[S V P>\tau]$. We define fairness index as the difference in service levels between the users with minimum (i.e. least-served user) and maximum (i.e. best-served user) service for a given policy over a given time frame.

\section{B. Simulation Setting: Adaptive Modulation \& Coding (AMC)}

Table I shows the standard parameters of the CDMA/HDR system which is based on AMC. It contains the standard combination of coding, modulation and packet size supported by a given data rate and $C / I$ to achieve $1 \%$ packet error rate in a $5-\mathrm{km}$ isolated cell with uniform distribution of users. Each user randomly picks a data rate control (DRC) index at the beginning of each scheduling interval from the standard twelve transmission modes shown in Table I. Once a DRC index is picked, the user requests, as per Table I, the corresponding data transmission rate from the serving base station. If a user is picked for service in a scheduling epoch then it transmits its HOL packet with length corresponding to its DRC index as per Table I over contiguous time slots. We set $w_{i}=1 / 2, w_{m}=1 / M$ and $d_{i}=1 \mathrm{sec}$ in the simulations.

\section{Results}

Figs 2 and 3 and Table II show the throughputs of the policies examined. We observe that for low $W$, i.e. $W \leq 150$ time slots, the achievable throughputs are in the decreasing order MaxSNR, OCASD, I-OCASD, PFS, M-LWDF, CASTI, TAOS-1, RR and then FIFO. However, as $W$ increases the throughput performance of OCASD, I-OCASD and PFS approach that of MaxSNR even for a small number of simultaneous users. This means that asymptotically (i.e. large user population $M$ and/or large scheduling sliding window $W$ ) MaxSNR, OCASD, I-OCASD and PFS can achieve the

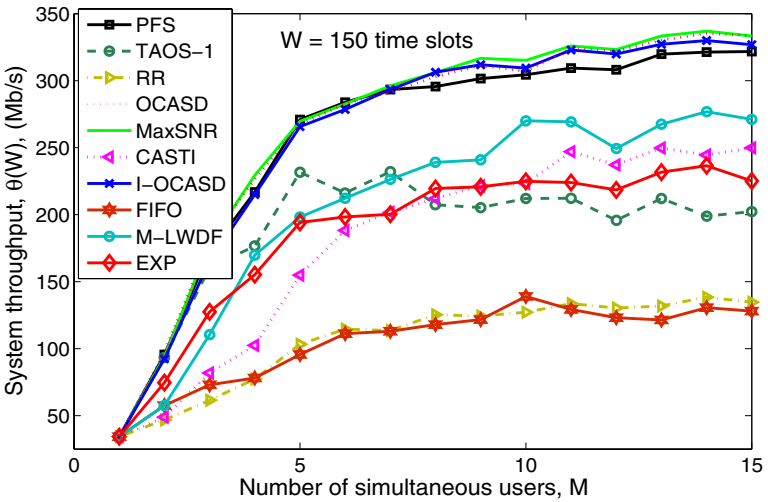

Fig. 2. System throughput for $W=150$ time slots.

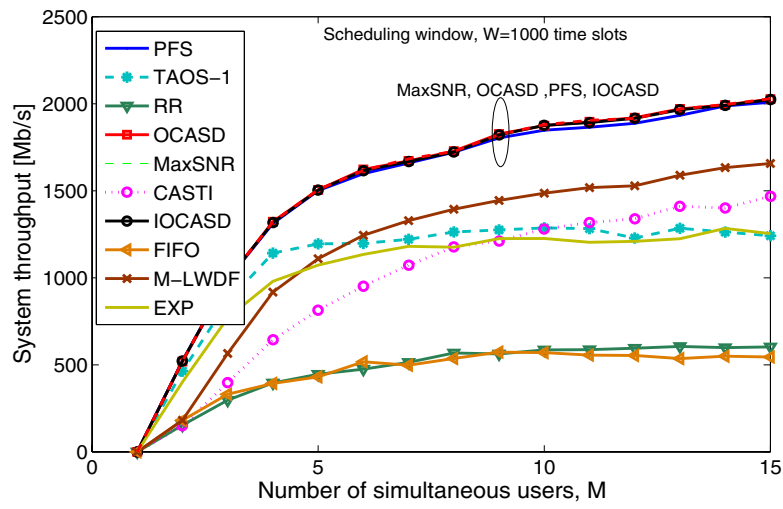

Fig. 3. System throughput for $W=1000$ time slots.

same system throughput. As we assumed that all users are backlogged continuously over the time frame simulated, FIFO queuing is expected to provide similar throughput performance as RR queuing.

Fig. 4 and Table II show the delay (user SVP) distribution of the ten policies discussed. Table II summarizes the lessons learnt from Fig. 4. Unlike the other policies, OCASD and I-OCASD provide queue isolation as well, making them attractive for multi-service networks. We observe that the EXP rule competes with FIFO in delay performance. Fig. 5 shows the user SVP, measured in time slots, averaged over number of simultaneous users $M$ versus $M$. The SVPs increase with $M$ to a certain value of $M$ and then begin to decrease. $\operatorname{Pr}[S V P>\tau]$ for PFS, I-OCASD, OCASD and MaxC/I fall sharply after $\tau \approx 1000$ time slots while that of TAOS1 remains at 0.5 until $\tau \approx 2,300$.

Fairness among the scheduling policies are discussed in Table II. The fairness indices in $\mathrm{Mb} / \mathrm{s}$ for a time frame of 2,500 time slots and 25 simultaneous users for the ten policies are respectively 3.5(TAOS-1), 8.0(PFS), 16.9(MLWDF), 18.4(EXP), 18.7(CASTI), 19.5(FIFO), 20.3(RR), 78.6(I-OCASD), 80.5(OCASD) and 89.0(MaxC/I). In terms of the amount of actual information transported, measured in 


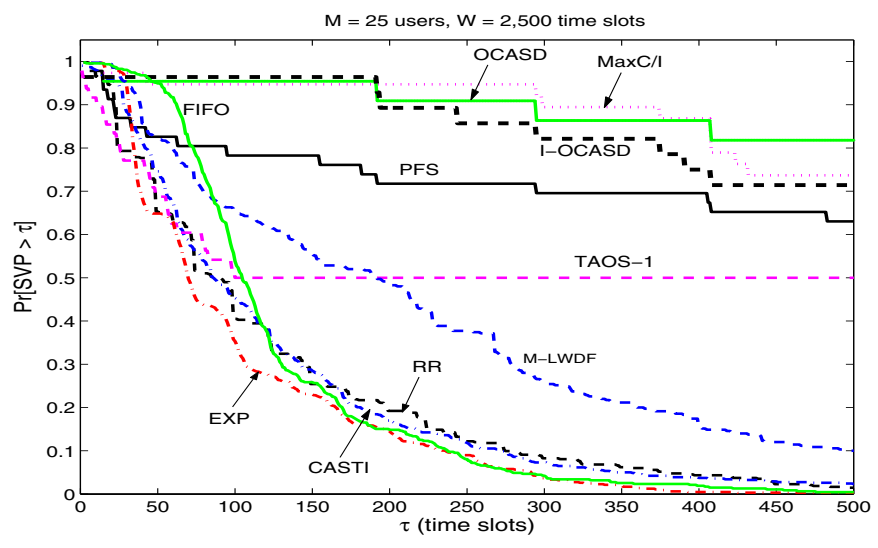

Fig. 4. Delay distribution: $\operatorname{Pr}[S V P>\tau]$ versus $\tau$.

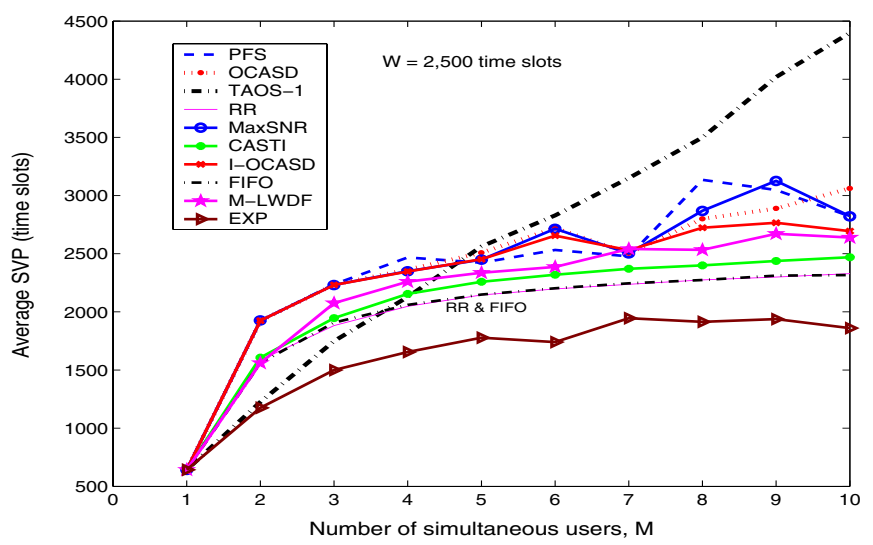

Fig. 5. Delay (average of SVP over $M$ ) versus $M$.

kBytes, the fairness indices are approximately 0.64(CASTI), 0.64(TAOS-1), 1.92(PFS), 3.2(EXP), 3.46(M-LWDF), 3.58(FIFO), 5.0(RR), 16.8(OCASD), 16.89(I-OCASD) and 19.07(MaxC/I). Hence, in terms of actual transported information bits, CASTI is the fairest policy while MaxC/I is the most unfair policy. However, in terms of cumulative data rate, TAOS-1 is the fairest policy followed by PFS. All the policies examined show good asymptotic fairness, i.e. when $W \rightarrow \infty$. In summary, we observe that RR is the fairest policy in wireless environment only in the number of service turns users/queues receive but not in actual throughput or service level. The exceptional fairness of TAOS-1 may have been affected by the parameter setting $F_{m}=L_{m}(t)$ in the simulations instead of interpreting $F_{m}$ as the remaining file size. Hence, due to its formulation, it should be fair both in bits/sec and bits.

\section{CONCLUSions}

This article has studied ten common scheduling schemes proposed for wireless networks using time-slotted transmissions. The results aid in the proper selection of an RRM scheme for a given network environment based on the performance metric(s) which needs to be emphasized. As an
TABLE II

COMPARISON OF POLICIES: $x<y$ MEANS POLICY $x$ PERFORMS BETTER THAN POLICY $y$ IN THE METRIC, $W=2,500$ SLOTS, $M=25$ USERS.

\begin{tabular}{lllll}
\hline $\begin{array}{l}\text { Scheduling } \\
\text { Policy }\end{array}$ & $\begin{array}{l}\text { Average } \\
\text { System } \\
\text { Throughput } \\
\text { (Mb/s) }\end{array}$ & $\begin{array}{l}\text { Average } \\
\text { System } \\
\text { Throughput } \\
\text { (kbytes) }\end{array}$ & $\begin{array}{l}\text { Average } \\
\text { Delay } \\
\text { Performance } \\
\text { Ordering }\end{array}$ & $\begin{array}{l}\text { Average } \\
\text { Fairness } \\
\text { Index } \\
\text { Ordering }\end{array}$ \\
\hline OCASD & 236.3965 & 49.3722 & 7 & 8 \\
I-OCASD & 235.9050 & 49.3056 & 6 & 9 \\
MaxC/I & 235.6347 & 49.2954 & 8 & 10 \\
PFS & 233.8775 & 48.9677 & 9 & 2 \\
M-LWDF & 187.1094 & 41.1187 & 5 & 5 \\
CASTI & 152.3482 & 35.5789 & 3 & 3 \\
TAOS-1 & 146.5974 & 30.6944 & 10 & 1 \\
EXP & 116.8835 & 28.9997 & 1 & 4 \\
RR & 24.5881 & 7.7926 & 4 & 7 \\
FIFO & 20.6083 & 6.8454 & 2 & 6 \\
\hline
\end{tabular}

example, assume that a CDMA/HDR network with time slot duration of $1.667 \mathrm{~ms}$ supports an application that requires the probabilistic delay guarantee of $\operatorname{Pr}[$ packet delay $\geq$ $250 \mathrm{~ms}] \leq 40 \%$. Figure 4 shows that only EXP, CASTI, RR and FIFO are candidate policies for the given situation, as 250 $\mathrm{ms}$ is equivalent to ca. 150 time slots and packet delay $\geq$ $S V P$. Among the four policies, CASTI is the optimal policy as it has the best system throughput and fairness index. Although there is some randomness in the simulation setting, the presented results are reasonably reliable.

\section{REFERENCES}

[1] A. Gyasi-Agyei, "Multiuser diversity based opportunistic scheduling for wireless data networks," IEEE Communications Letters, vol. 9, no. 7, pp. 670-672, July 2005.

[2] —, "OCASD-channel-aware scheduling for multi-service wireless IP networks," in Proc. Australian Telecommunications, and Networks Applications Conference (ATNAC), Sydney, December 2004, pp. 582-589. [Online]. Available: http://www.titr.uow.edu.au/atnac/Proceedings/abstract.pdf

[3] — "Mobile IP-DECT internetworking architecture supporting IMT2000 applications," IEEE Network, vol. 15, no. 6, pp. 10-22, Nov/Dec 2001.

[4] T. Bonald, "Flow-level performance analysis of some opportunistic scheduling algorithms," European Transactions on Telecommunications, vol. 16, pp. 65-75, 2005.

[5] A. Gyasi-Agyei, "I-OCASD: A multiservice cross-layer scheduler for packetized wireless networks," in Submitted to IEEE Vehicular Technology Conference (VTC06), Melbourne, Australia, 2006.

[6] — " $\mathrm{BL}^{2} \mathrm{xF}-$ channel state-dependent scheduling algorithms for wireless IP networks," in Proc. IEEE Int. Conf. on Networks (ICON'03), Sydney, September 2003, pp. 623-628.

[7] A. Jalali, R. Padovani, and R. Pankaj, "Data throughput of CDMAHDR: a high efficiency-high data rate personal communication wireless system," in Proc. IEEE VTC 2000-Spring, Tokyo, Japan, May 2000, pp. 1854-1858.

[8] P. Viswanath, D. N. C. Tse, and R. Laroia, "Opportunistic beamforming using dumb antennas," IEEE Trans. Inform. Theory, vol. 48, no. 6, pp. 1277-1294, Jun 2002.

[9] M. Andrews, K. Kumaran, K. Ramanan, A. Stolyar, R. Vijayakumar, and P. Whiting, "CDMA data QoS scheduling on the forward link with variable channel conditions," Bell Labs Technical Report preprint, April 2000.

[10] S. Shakkottai and A. Stolyar, "Scheduling algorithms for a mixture of real-time and non-real-time data in HDR," in Proc. 17th Int. Teletraffic Congress (ITC-17), Salvador de Bahia, Brazil, September 2001.

[11] M. Hu, J. Zhang, and J. Sadowsky, "Traffic aided opportunistic scheduling for wireless networks: algorithms and performance bounds," Computer Networks J., 2005. 